・物种概念专题・

\title{
浅析物种概念的演变历史
}

\author{
谢 平 $^{*}$ \\ (中国科学院水生生物研究所, 武汉 430072)
}

\begin{abstract}
摘要: 本文是一篇关于物种概念演变的简述。生物学家用不同的方法或标准划分物种, 就形成了不同的物种概念, 如生物学物种、形态学物种、生态学物种、进化物种、系统发生或支序物种, 或它们的组合, 等等。它们都揭示 了物种属性的特定侧面, 都是不同物种客观存在的真实反映, 但都无法令所有人满意。对真核生物来说, 无论它们 在形态上的差别有多大, 生殖隔离(不能产生可育的后代)应该是两个群体能否真正分化成不同物种的关键, 这种 隔离机制可以是地理的、行为的或其他方式; 而生殖隔离总会伴随着一些形态或遗传上的变化, 虽然这些特征可 能与生殖隔离本身并无多大关系, 但往往成为分类学家或分子进化生物学家区分种的依据,对已经灭绝的化石物 种来说, 生殖隔离的物种划分方式就无能为力了。如何准确定义一个物种依然充满着矛盾, 因为基于生殖隔离的 物种概念不实用，而实用的物种概念(如形态学物种)又被认为是人为的。
\end{abstract}

关键词: 物种概念; 生物学物种; 形态学物种; 生殖隔离

\section{A brief review on the historical changes in the concept of species}

\section{Ping Xie}

Institute of Hydrobiology, Chinese Academy of Sciences, Wuhan 430072

\begin{abstract}
This is a mini-review on the historical changes in the concept of species. Biologists use different methods or criteria to discriminate species, leading to the formation of different species concepts, e.g. biological species, morphospecies, ecological species, evolutionary species, phylogenetic/cladistic species, or their combinations. These concepts respectively reveal a specific profile of the species' attributes, as well as reflecting the objective existence of these creatures as different species, but not being satisfied with everyone. For eukaryotes, reproductive isolation (incapable of reproducing fertile offspring) should be the key for two populations to differentiate into two different species, no matter how much they differ morphologically. The mechanisms underlying such isolation might be geographical, behavioral, or otherwise. Reproductive isolation is certainly accompanied by some morphological or genetic changes that are often used as criteria by taxonomists or molecular evolutionary biologists to distinguish species, although these attributes may not be associated with reproductive isolation itself. Extinct species known only from fossils are impossible to be classified taxonomically according to reproductive isolation. The exact definition of the term "species" is still controversial, as a species concept based on reproductive isolation is usually not applicable, but a usable species definition (e.g. morphospecies) is regarded to be artificial.
\end{abstract}

Key words: species concept; biological species; morphospecies; reproductive isolation

科学术语“物种(species)”来源于拉丁文中表示 性状或现象的一个词(Offenberger, 1999)。物种是生 命存在与繁衍的基本单元，它既有相对的稳定性， 又有绝对的变化性。如果它亘古不变, 便没有生物 界的进化, 但它也不能转瞬即逝。只有当人们试图
对生物界进行分门别类时，才不得不思考如何对物 种的内涵进行合理的界定。

\section{1 关于物种划分的困惑}

关于物种, 达尔文(Charles Robert Darwin,

收稿日期: 2016-08-29; 接受日期: 2016-09-02

* 通讯作者 Author for correspondence. E-mail: xieping@ihb.ac.cn 
1809-1882)曾说: “没有一项定义能使一切博物学者 都满意; 然而各个博物学者在谈到物种的时候, 都 能够模糊地知道它是什么意思……。关于 ‘变种'这 个名词, 几乎也是同样地难下定义……。华莱士认 为动物可以分为四类: 即变异类型、地方类型、地 理族即地理亚种以及真正的、具有代表性的物种” (Darwin, 1872)。

Dobzhansky (1951)认为: “动物和植物分类学家 利用有机体变异的不连续性来给生物进行分类和 定名……, 分类的范畴是学者为了自己的方便而设 计的结构; 在这种意义上, 它们有时候被认为是“武 断的’或“主观的'…… 但是有些分类的类群, 特别 是种和属, 分类学家通过精心努力, 而使它们和一 些大小不同的孟德尔式群体尽可能紧密符合”。

Barton 等(2007)指出: “人们普遍承认种的划分 是真实的。而通常存在争议的是较高层次的分类阶 元, 如属或科。类似地, 人们往往不清楚一个种内 的不同形式是否应该分为地理种或亚种, 却通常很 清楚生活在任何一个地方的生物体应该怎样归到 种里”。

一些唯名论(nominalism) 者则宣称, 物种只是 人们给特定类群的生灵所取的名字而已, 人们给物 种所画的线也不代表任何生物学的基本分界点, 因 此, 物种仅存在于人的大脑中, 不过是一种命名抽 象或精神抽象而已(Gregg，1950)。而实用主义 (pragmatism) 者认为, 物种在概念上是真实的, 为了 我们的便利与实际应用而存在(Dupré, 2001)。

\section{2 强调不变性的物种概念}

物种被视为特征明显的、不变的实体, 物种由 关键的性状确定, 通过这些特征可以自动划归类 似的个体。这种观点的起源可以远溯到柏拉图 (Plato, 约公元前 427 年至公元前 347 年)的理念论 哲学, 按照这种哲学, 物种被认为是一种比构成 它的个体更深层次的存在。确定物种本质的是理念 化的形式或结构, 而不是任何一个个体的表面性 状(Bowler, 1989)。可以认为, 柏拉图强调了物种的 绝对不变性, 它由本质(essence)所决定, 就像化学 元素一样。如果按现代的知识来理解, 柏拉图所说 的理念化的形式或结构就是由 DNA 所编码的形式 或结构。

\section{3 强调变异性的物种概念}

\section{1 拉马克一一生物之间存在不可分割的连续性}

拉马克(Jean-Baptiste Lamarck, 1744-1829)认为 物种只是一个人为的概念, 事实上并不存在。根据 他的用进废退理论, 所有生物应该存在一种不可分 割的连续性，如果收集足够的标本来填补物种之间 形态上的缺失, 就可以看出生物界是一个由一连串 连续的过渡形态联结在一起的整体。既然所有物种 都处在一个连续变化的过程之中, 也就无所谓物种 的灭绝, 有的只是从一种形态转变为另一种形态 (Shi, 2010)。显然, 拉马克过分强调了物种的变异性 而忽视了物种的相对稳定性。

\section{2 达尔文一一物种是一个在极长的时期内一面 改变、一面分化的产物}

达尔文拒绝为物种下一个定义，他在《物种起 源》中写道: “在物种和变种这些名词的定义还没有 得到普遍承认之前，就来讨论什么应该成为物种， 什么应该成为变种，乃是徒劳无益的……有些博 物学家认为亚种已很接近物种, 但还没有完全达到 物种一级; 在物种和亚种之间, 的确还没有划出过 明确的界限; 此外, 在亚种和显著的变种之间, 在 较不显著的变种和个体差异之间, 也未曾划出过明 确的界限…… 如果一个变种很繁盛, 而超过了亲 种的数目, 那么, 它就会被列为物种, 而亲种就被 当作变种了; 或者它会被亲种消灭而代替; 或者两 者并存, 都被列为独立的物种……我认为物种这 个名词是为了便利而任意加于一群彼此非常类似 的个体的，它和变种这个名词在本质上并没有区别， 变种是指区别较少而彷得较多的类型。还有, 对变 种这个名词和个体的差别比较, 也是为了便利而任 意取用的” (Darwin, 1872)。

对达尔文来说，一旦有机生命被看作是一个在 极长的时期内一面改变、一面分化的产物, “物种” 这个概念就成了相当任意的了。从进化的观点来看, 它仅仅是为了较方便地指代那些由比较紧密联系 在一起的个体形成的群体而已，虽然这对于分类和 形态描述的目的来说可能是必要的(Magner, 1994)。

达尔文认为: “物种只是特征非常显著的、稳定 的变种, 而且每一物种首先是作为变种而存在” (Darwin, 1872)。达尔文如此的物种观正是他内心矛 
盾的真实写照。这样的见解既有一定的道理, 也存 在缺憾, 反映了达尔文过分强调物种变异性的倾 向。对后来的生物学家来说, 它带来了人们对物种 不确定性的认知恐慌。其实, 没有变种就不可能有 新物种的诞生, 如果物种之间在形态等特征上没有 间断性, 那就不可能有真正的物种存在了。

\section{4 主张既有稳定性也有变异性的物种概念}

\section{1 赫伯特一一物种是比较高级和比较稳定的 变化}

赫伯特(R. W. Herbert)在 1822年Horticultural Transactions 第四卷和他于 1837 年出版的 Amaryllidaceae (石蒜科)一书中宣称, 园艺试验不可辩驳 地证明了植物学上的物种不过是比较高级和比较 稳定的变化而已。他认为动物亦如此, 并相信每一 个属的单一物种都是在原来可塑性很大的情况下 被创造出来的, 这些物种主要由于杂交也由于变异 而产生现存的一切物种(Darwin, 1872)。

\section{2 居维叶一一物种在一定限度内永远存在下去, 变种是物种偶然的再细分}

居维叶(Georges Cuvie, 1769-1832)将物种定义 为“从原初形成开始, 使它们在一定的限度内永远 存在下去的某种形式”, 变种的存在“是物种偶然再 细分的结果”。他还认为对物种的定义应该超出形 态学的特点, 并且应该包括遗传的或历史的成份。 他认为: 由于生殖是确定变种扩散范围的唯一方法, 物种应当定义为这样一些个体的再结合, 它们中一 些个体是其他个体的后裔; 或者是共同亲本的后裔; 或者是另外一些个体的后裔, 这些个体与它们的相 似程度与它们相互间的相似程度相当。他意识到检 验性的交配实验并不是在任何情况下都能办到的 (Magner, 1994)。

\section{3 陈世骧一一又变又不变的物种概念}

我国著名的昆虫学家和进化分类学家陈世骧 是这样定义的: 物种是由种群所组成的生殖单元, 在自然界中占据有一定的生境, 在谱系上归属于一 定的分支。他认为物种既变也不变。之所以说物种 是变的, 是因为每一物种都有自己的特征, 没有两 个物种完全相同; 但物种又是不变的, 这是因为每 一物种都保持有一系列祖传的特征, 籍此反映其进 化历史, 决定其分类的隶属。他认为这是分类系统 之所以反映进化历史的根本原因, 而如何反映进化
历史，关键在于特征分析(陈世骧，1983)。

\section{5 强调生殖隔离的物种概念}

\section{1 雷———个物种可通过种子繁殖而永远延续}

英国博物学家雷(John Ray, 1627-1705)是第一 个把物种作为分类单元, 建立了适合于动植物分类 系统的博物学家, 他在 Historia Plantarum 中描述的 植物分类是迈向现代分类学的重要一步。雷认为通 过种子可以产生相同后代的植物应被归为一个种, 即定义一个物种必须以繁殖试验为基础, 1686 年他 写道: “经过长期而大量的观察后, 我相信在确定一 个物种时，除了应该把通过种子繁殖而使之永远延 续的特点作为标准外，没有其他更合适的标准。因 此不管一个个体或一个物种发生了什么样的变化, 如果它们是由同一棵植物或同样的植物种子发芽 生长起来的, 那么这些变化只能称作是偶然的变异 而不能作为区分一个物种的标志”, 即不重要的变 化不应成为鉴定种的依据。他认为, 尽管所有的物 种都是上帝创造的成对个体的后代，但在类型上还 是具有某些可变性的, 物种的性状并不绝对固定不 变(Magner, 1994)。这应该就是被迈尔称之为生物学 物种(biological species concept, BSC)的最早表述。 虽然生殖隔离的物种概念被分类学家批评为可操 作性不强，却得到育种学家的青棟。

\section{2 布丰一一生殖隔离是鉴别物种的重要标准}

法国博物学家布丰(Georges Louis Leclere de Buffon, 1707-1788)从骡子的繁殖行为认识到物种 在自然界中的客观存在。他认为, 物种是这样一些 动物的组合，它们之间是可以通过杂交来产生可育 的后代的，而不会产生不育的杂种。他将此视为一 种检验某些个体是否属于同一物种的方法。这与雷 的见解相似。布丰认为, 物种确实是自然界惟有的 存在，它像自然本身一样古老而久远，而“一个个 体, 无论它属于哪种物种, 在宇宙中只不过是无”, 一个物种是“一个独立于数, 独立于时间的整体, 一个永远生存, 永远同一的整体……” (Lovejoy, 1982; Magner, 1994)。布丰认为, 如果我们能证明一 群个体属于一个由生殖维持的群体, 那么我们就可 以确定它为一个真正的物种(Bowler, 1989)。

其实，布丰对物种的观点也经历了变化的过 程。他在《自然史》一书的第一卷中, 否认物种是 真正的实体, 认为物种以及由物种组成的属只不过 
是分类学家想象出来的产物, 实际存在的只是个体, 而且我们有时可以发现两个所谓区别明显的物种 之间的类型。然而, 之后的两卷中, 他又写道, 物种 是固定不变的、区别明显的实体, 他在以后一直秉 持这种观点, 虽然略有改变(Bowler, 1989)。

\section{3 沃尔什一一不能自由杂交的即为物种}

据达尔文记载, 美国昆虫学者沃尔什 (Benjamin Dann Walsh, 1808-1869) “把那些假定可 以自由杂交的类型列为变种; 把那些看来已经失去 这种能力的列为物种” (Darwin, 1872)。这似乎也是 将生殖隔离作为物种划分的标准。

\section{4 迈尔——生物学物种概念}

德裔美国进化生物学与分类学家迈尔(Ernst Walter Mayr, 1904-2005)提出了所谓生物学物种概 念, 认为物种是由相互配育的自然群体组成的类群, 这些类群与其他类群之间存在着生殖隔离, 即一个 物种就是一个生殖共同体(Mayr, 2001)。其实, 用生 殖隔离来区分不同物种的思想在数世纪之前就已 存在, 如 17 世纪的雷, 18 世纪的布丰等。但是, 在 很多情况下, 群体之间能否相互配育在技术上是难 以确认的, 分类学家不得不主要根据形态学的差异 程度来进行决断。

地理隔离是造成生殖隔离的重要因素之一, 达 尔文早就注意到这一点。迈尔曾在 1942 年指出, 如 果某一种群和它的亲本物种被地理隔离并在隔离 阶段获得了当外界障碍物消失时仍能促进或保证 生殖隔离的有关性状, 那么就形成了新的物种 (Mayr, 1982)。地理隔离的物种形成方式也称之为异 域成种，但自然界中还存在大量的同域成种现象。

当然, 生物学物种概念只适用于进行有性生殖 的生物。Barton 等(2007)指出, 如果我们坚持严格的 生物种概念, 那么一个无性繁殖的群体里每个成员 都是一个分离的“种”, 因为不和任何一个其他个体 进行杂交……。尽管许多生物学家用生殖隔离来定 义物种形成, 但是实际上物种的定义通常是基于形 态的。

\section{6 基于群体遗传学的物种概念}

\section{1 杜布赞斯基一一种是最概括性的孟德尔式 群体}

Dobzhansky (1951)认为, 物种不是一个静态单 位, 而只是进化分歧过程中的一个阶段。他认为,
种就是一些群体的类群，其间的基因交换，在自然 界是受一种或几种生殖隔离机制的组合所限制或 阻止。总而言之, 种是最概括的孟德尔式群体…… 孟德尔式群体之间在遗传上的不连续性, 因生殖隔 离而变得固定下来……。这种阶段的到达, 就是物 种分化的来临。

他指出: “在孟德尔式群体间，杂交繁殖、基因 互换以及生殖隔离的有无, 只能借助于实验遗传学 和生态学的方法才能直接加以研究。分类学家很少 能运用这些方法来描述或研究种”。

\section{2 巴顿等一一一个物种形成一个独立的基因库}

英国进化遗传学家巴顿(Nicholas H. Barton)等 指出: “一个生物种形成单独一个基因库, 所以一个 个体中产生的偏好等位基因最终可以扩散到整个 物种。原则上，通过重组，任何该等位基因的复合 体都可以在一个生物种内部产生。相反, 不同生物 种各自独立进化, 在这种意义上, 一个生物种遗传 变异对另一个生物种的进化没有贡献” (Barton et al, 2007)。但是, 从生态的视角来看, 我们不能排除一 个物种的遗传变异对另一个物种的进化作出贡献 的可能性。

\section{7 基于基因差异的物种概念}

一些学者怀疑基于形态学特征的物种划分, 认 为基于 DNA 序列差异的物种分类更为可靠。譬如, Barton 等(2007)认为: “分子差异似乎是更客观的标 准; 可以用 DNA 序列将个体划到种里。对于不可 培养的微生物, 这种方法是更好的选择。很多研究 表明: 如果一个样本的核糖体 RNA 序列与另外一 个样本的另外一条核糖体 RNA 序列的一致性低于 $97 \%$, 那么它们是不同的种”。但问题是为何这个标 准是某个值，譬如 $97 \%$ ？笔者认为，将某个特定的 差异值作为不同物种的分界线同样是一种颇为主 观的做法，比基于形态的分类标准有时可能更糟， 但对既难以依赖形态也难以依赖生殖的原核生物 来说, 或许是一种无奈的选择。原核生物或许就是 若干个大的基因库，而基因在我们现在认为的所谓 亲缘关系较远的类群之间亦能自由流动。

英国进化生物学家道金斯(Richard Dawkins)曾 说，两个个体同种(conspecific)的充分必要条件是, 它们有同样数目的染色体, 以及每个染色体具有同 样数目的核苷酸(Dawkins，1986)。但事实上，有些 
物种的不同种群之间, 染色体数目是高度变异的, 而种群之间仍然可以交配; 植物的多倍性 (polyploidy)现象极为普遍。

\section{8 其他物种概念}

还有一些其他的物种概念, 例如: (1)强调进化 的物种概念(如进化物种、接替物种、时间物种); (2) 强调系统发生的物种概念(如系统发生物种、支序物 种、节点间物种); (3)强调生态的物种概念; (4)强调 形态的物种概念(如形态学物种、分类学物种、形态 -地理学物种、表征物种) (洪德元, 2016)。依笔者之 见, 所谓的进化物种概念不过是对物种进化或系统 发生特性、途径或结果的描述, 强调生态的物种概 念更难以成为物种的判别标准, 而自林奈以来的形 态学物种是分类学的产物, 是最具可操作性的物种 概念。由于形态的差异不一定与生殖隔离直接相关, 因此基于形态差异的物种划分常常被批评为分类 学家的主观性, 这亦因为绝大多数物种的分类都无 法通过生殖隔离实验来确认。

洪德元先生提出了“形态-生物学物种概念”, 试图将形态、生物学、遗传学、进化等物种概念予 以整合, 认为一个物种应该能够反映下述属性: (1) 体现物种内的所有成员共享一个基因库, 个体间和 居群间存在基因流, 而物种之间由于隔离(也包括 地理隔离和生态隔离), 不发生基因交换; (2)一个独 立的谱系, 与别的谱系分别进化; (3)形成至少有两 个共有衍征的最小的单系群; (4)与别的物种在生态 位上有分化; (5)有独立的地理分布(洪德元, 2016)。 其实, 物种的同域分化现象也不罕见, 譬如, 在同 一个湖泊中, 通过生态位细分而形成新的近缘物种 (陈宜瑜等, 1982)。

\section{9 结语}

物种概念可追溯至古希腊的哲学家, 关于物种 概念的哲学争议延续至今。生物学家用不同的方法 或标准划分物种, 就形成了不同的物种概念, 如生 物学物种 (biological species)、形态学物种 (morphospecies)、生态学物种(ecological species)、 系统发生或支序物种(phylogenetic or cladistic species), 或它们的组合, 等等。它们都揭示了物种属性 的特定侧面, 都是物种客观存在的真实反映, 但都 无法令所有人满意。
依笔者之见, 对真核生物来说, 无论它们在形 态上的差别有多大, 生殖隔离应该是两个群体能否 真正分化成不同物种(成种， speciation)的关键，这 种隔离机制可以是地理的、行为的或其他方式; 而 生殖隔离总会伴随着一些形态或遗传上的变化, 虽 然这些特征可能与生殖隔离本身并无多大关系，但 往往成为分类学家或分子进化生物学家区分种的 依据。另一方面, 对化石物种来说, 无法验证是否 存在生殖隔离。因此, 古生物学家不得不寻求其他 方面的证据, 如结构的相似性等, 这样, 所判定的 化石种(通常停留于较高的分类单元如科)就具有更 多的或然性(谢平, 2016)。

迄今为止, 生物学界在物种定义的问题上尚未 达成一致，还没有一个被公认的能涵盖所有生物类 群的物种概念, 主要原因是在物种划分的标准上难 以达成统一。譬如, 如果以生殖隔离来划分, 而生 殖方式又太复杂, 因此生物学物种概念仅适合于有 性生殖的物种(即便如此, 大多数物种之间的生殖 隔离仍难以在实践中确认), 但对像细菌这类进行 无性生殖的物种却无能为力, 对已经灭绝的化石物 种也是无法适用的(谢平, 2016)。

\section{参考文献}

Barton NH, Briggs DEG, Eisen JA, Goldstein DB, Patel NH (2007) Evolution. Cold Spring Harbor Laboratory Press, New York.

Bowler PJ (1989) Evolution: the History of an Idea. University of California Press, Berkeley.

Chen SX (1983) Species concept and taxonomic principles. Science in China Series B, 4, 315-320. (in Chinese with English abstract) [陈世骧 (1983) 物种概念与分类原理. 中国科学B辑, 4, 315-320.]

Chen YY, Zhang W, Huang SY (1982) Speciation in Schizothoracid fishes of Lake Lugu. Acta Zoologica Sinica, 28, 217-225. (in Chinese with English abstract) [陈宜瑜, 张卫, 黄顺友 (1982) 沪沽湖裂腹鱼类的物种形成. 动物学报, 28, 217-225.]

Darwin C (1872) On the Origin of Species by Means of Natural Selection, or the Preservation of Favoured Races in the Struggle for Life, 6th edn. John Murry, London.

Dawkins R (1986) The Blind Watchmaker. Norton \& Company, Inc., New York.

Dobzhansky T (1951) Genetics and the Origin of Species. Columbia University Press, New York and London.

Dupré J (2001) In defence of classification. Studies in History and Philosophy of Biological and Biomedical Sciences, 32, 203-219.

Gregg JR (1950) Taxonomy, language and reality. The Ameri- 
can Naturalist, 84, 419-435.

Hong DY (2016) Biodiversity pursuits need a scientific and operative species concept. Biodiversity Science, 24, 979999. (in Chinese with English abstract) [洪德元 (2016) 生 物多样性事业需要科学、可操作的物种概念. 生物多样 性, 24, 979-999.]

Lovejoy A (1982) The Great Chain of Being, a Study of the History of an Idea. Harvard University Press, Cambridge.

Magner LN (1994) A History of the Life Science. Marcel Dekker, New York.

Mayr E (1982) The Growth of Biological Thought-Diversity Evolution \& Inheritance. Belknap Press, Cambridge.
Mayr E (2001) What Evolution Is? Basic Books, New York. Offenberger M (1999) Von Nautilus und Sapiens. Einführung in die Evolutionstheorie. Deutscher Taschenbuch Verlag GmbH \& Co. KG, Munich, Germany.

Shi J (2010) Evolution! Evolution? The War Behind Darwin. Liaoning Education Press, Shenyang. (in Chinese) [史钧 (2010) 进化! 进化? 达尔文背后的战争. 辽宁教育出版 社, 沈阳.]

Xie P (2016) Critical Reviews and Reconstruction of Evolutionary Theories. Science Press, Beijing. (in Chinese) [谢平 (2016) 进化理论之审读与重塑. 科学出版社, 北京.] 